\title{
QUALIDADE FITOSSANITÁRIA DAS CASCAS DE AROEIRA \\ (Myrocroduon urundeuva Allemão) E CATUABA (Erythroxylum vacciniifolium Mart.) COMERCIALIZADAS EM FEIRAS MUNICIPAIS DE IMPERATRIZ, MA
}

\author{
Ricardo Vieira Silva'; Gustavo de Andrade Bezerra ${ }^{2}$; Ivaneide de Oliveira \\ Nascimento $^{3}$; Thatyane Pereira de Sousa ${ }^{4}$; Paulo Henrique Aragão Catunda ${ }^{5}$; Karuane \\ Saturnino da Silva Araújo6.

\footnotetext{
1Universidade Estadual do Maranhão. Imperatriz, Maranhão, Brasil.rickvieira_5@ hotmail.com

${ }^{2}$ Universidade Estadual do Maranhão. Imperatriz, Maranhão, Brasil. guandrade.b@ gmail.com ${ }^{4}$ Universidade Federal Rural da Amazônia. Belém, Pará, Brasil. thatyane_@ @otmail.com

${ }^{5}$ Universidade Estadual do Maranhão. Imperatriz, Maranhão, Brasil. phuema.cesi@gmail.com

${ }^{6}$ Universidade Estadual do Maranhão. Imperatriz, Maranhão, Brasil. karuane@gmail.com
} \\ ${ }^{3}$ Universidade Estadual do Maranhão. Imperatriz, Maranhão, Brasil. ivaneide_agro@yahoo.com.br
}

\begin{abstract}
RESUMO: O uso de plantas medicinais tornou-se muito representativo, pelo fato de ser comercializada como medicamento alternativo para a cura de determinadas doenças. Mesmo com o avanço da indústria farmacêutica e a evolução da ciência de medicamentos, o uso dessas plantas para a extração de substância ativa e material vegetativo ainda é indispensável. O presente trabalho objetivou realizar a análise fitossanitária em cascas de aroeira (Myrocroduon urundeuva Allemão) e catuaba (Erythroxylum vacciniifolium Mart.), comercializadas em Imperatriz, MA. Realizou-se a coleta das cascas de aroeira e catuaba, em três feiras do município, estas foram acondicionadas em sacos plásticos e encaminhadas ao Laboratório de Fitopatologia, Microbiologia e Alimentos da Universidade Estadual do Maranhão - Centro de Estudos Superiores de Imperatriz, onde as cascas passaram pelo processo de desinfestação superficial com álcool 70\%, hipoclorito de sódio a 2 e água destilada e, foram inoculadas em meio padrão de Batata, Dextrose e Ágar (BDA) e, mantidas em laboratório na condições controladas de temperatura entre $25-30^{\circ} \mathrm{C}$, por sete dias. $\mathrm{Na}$ análise de incidência fúngica foram identificados: Aspergillus flavus, Aspergillus niger, Fusarium moniliforme, Fusarium solani, Penicillium italicum e Rhizopus sp. Contudo, os gêneros Aspergillus sp. e Fusarium sp. foram os de maior ocorrência.
\end{abstract}

PALAVRAS-CHAVE: fungos, micotoxinas, plantas medicinais.

\section{PLANT HEALTH QUALITY OF PEEL OF AROEIRA (Myrocroduon urundeuva Allemão) AND CATUABA (Erythroxylum vacciniifolium Mart.) COMMERCIALLY FAIR MUNICIPAL EMPRESS, MA}

\begin{abstract}
The use of medicinal plants has become very representative, because it is marketed as an alternative medicine to cure certain diseases. Even with the advancement of the pharmaceutical industry and the evolution of the science of medicine, the use of these plants for the extraction of the active substance and plant material is still indispensable. This study aimed to perform the analysis in plant husks mastic (Myrocroduon urundeuva Allemão) and catuaba (Erythroxylum vacciniifolium Mart.), marketed in Imperatriz, MA. Held collection of mastic and catuaba bark, three county fairs, these were packaged in plastic bags and sent to the Laboratory of Plant Pathology, Microbiology and Food at the State University of Maranhão - Center of Study Top Empress, where the shells through the process of surface
\end{abstract}


disinfection with $70 \%$ alcohol, sodium hypochlorite and distilled water and 2 were inoculated in standard potato, and dextrose agar (PDA), and maintained in the laboratory under controlled temperature between $25-30^{\circ} \mathrm{C}$ for seven days. In the analysis of fungal incidence were identified: Aspergillus flavus, Aspergillus niger, Fusarium moniliforme, Fusarium solani, Penicillium italicum and Rhizopus sp. However, Aspergillus sp. and Fusarium sp. were the most frequent.

KEYWORDS: fungi, medicinal plants, mycotoxins.

As plantas medicinais, na atualidade, são de primordial importância para o tratamento de doenças sendo usada como remédio, substituindo assim, os princípios ativos desenvolvidos pelas indústrias farmacêuticas. Entretanto, as plantas são utilizadas de forma incorreta, sem o devido tratamento sanitário, pondo em risco a saúde humana (CELESTE et al., 2004).

Ainda hoje nas regiões mais pobres do Brasil e até mesmo nas grandes cidades, plantas medicinais são comercializadas em feiras livres, mercados populares e encontradas em quintais residenciais (ANGRA et al., 2007).

O material vegetal usado merece atenção especial quanto ao manuseio, alguns autores discutem a falta de preocupação com estes cuidados que podem levar à deterioração, tais como, a degradação de substâncias ativas, contaminação por parasitas, roedores, fungos e outros microrganismos (SILVA et al., 1995).

Esses organismos apresentam substâncias tóxicas, tais como as micotoxinas liberadas por fungos filamentosos, são toxinas que põe em risco a saúde humana podendo levar até a morte. Micotoxinas são metabólitos secundários, aparentemente sem qualquer função no metabolismo normal dos fungos (PRADO, 2008). Elas são produzidas, ainda que não exclusivamente, à medida que o fungo atinge a maturidade (FREIRE et al., 2007).

O objetivo deste trabalho foi realizar a análise fitossanitária em cascas de aroeira (Myrocroduon urundeuva Allemão) e catuaba (Erythroxylum vacciniifolium Mart.), comercializados em diferentes feiras livres de Imperatriz, MA.

O trabalho foi conduzido no Laboratório de Fitopatologia, Microbiologia e Alimentos do Centro de Estudos Superiores de Imperatriz da Universidade Estadual do Maranhão. O material vegetal foi adquirido nas feiras municipais de Imperatriz, levando em consideração na análise somente as cascas.

As cascas coletadas para análise foram de duas espécies: aroeira e catuaba. As quais $\operatorname{logo}$ após terem sido adquiridas, 
foram acondicionadas em sacos plásticos e identificas com o nome popular no local de aquisição.

No laboratório, o procedimento de isolamento de fungos foi realizado utilizando duas metodologias: uma com desinfestação superficial e outra com desinfestação superficial em ambiente asséptico.

Foram retiradas 40 (quarenta) fragmentos, com aproximadamente $0,5 \mathrm{~cm}^{2}$ do material vegetal, onde 20 (vinte) fragmentos de material vegetal foram expostos a uma sequência de lavagens e o restante foi utilizado no procedimento sem desinfestação.

O procedimento de desinfestação iniciou-se com uma lavagem que ocorreu em álcool a 70\% durante 30 segundos, a segunda em hipoclorito de sódio $(\mathrm{NaOCl})$ a $2 \%$ durante 20 segundos, seguida de três lavagens com água destilada por 20 segundos cada. Após a desinfecção os fragmentos foram expostos a papel de filtro para a retirada do excesso de água. Em seguida foi realizado o plaqueamento. Em cada procedimento foram utilizados 20 fragmentos dispostos em cinco placas de Petri, contendo meio padrão composto de Batata, Dextrose e Ágar (BDA), com 4 fragmentos em cada placa.

As placas foram devidamente vedadas e mantidas em condições de laboratório na temperatura de $25-30^{\circ} \mathrm{C}$, por 7 (sete) dias.
Após esse período, foram preparadas lâminas com azul de metileno e em seguida observadas em microscópio óptico.

A identificação dos fungos isolados foi realizada por comparação das características estruturais reprodutivas e de infecção de cada isolado, como descritas nas chaves de identificação de fungos, segundo Barnett e Hunter (1998), disponíveis na literatura especializada.

Nas análises laboratoriais, o material vegetal usado, as cascas de aroeira e catuaba apresentaram uma infecção fúngica significativa, onde foi possível observar as seguintes espécies de fungos: Aspergillus niger, Aspergillus flavus, Fusarium moniliforme, Fusarium solani, Penicillium italicum e Rhizopus sp. (Figura $1)$.

Nas análises das espécies do material vegetal, os gêneros Fusarium sp. e Aspergillus sp. foram os de maior infestação nas amostras.

Observando as análises e os resultados obtidos, constatou-se que no material vegetal da aroeira houve maior infestação fúngica, apresentando quatro espécies (Aspergillus niger, Aspergillus flavus, Fusarium moniliforme e Rhizopus sp.) com maior incidência fúngica em relação a catuaba.

O gênero Fusarium sp. foi o de maior infestação na espécie da catuaba, com percentual representativo de $40,48 \%$, 
seguido do Aspergillus sp. com 35,71\%. Espécies de Fusarium incluem importantes fitopatógenos, causadores de murchas, podridões, morte de plântulas, aborto de flores, podridões de armazenamento e outras doenças. De ocorrência cosmopolita, frequentemente estão associados com sementes. Algumas espécies são produtoras de importantes micotoxinas (CAB, 2013).

Figura 1. Fungos fitopatogênicos encontrados nas análises laboratoriais de material vegetal (cascas de aroeira e catuaba) coletadas em feiras municipais de Imperatriz, MA.

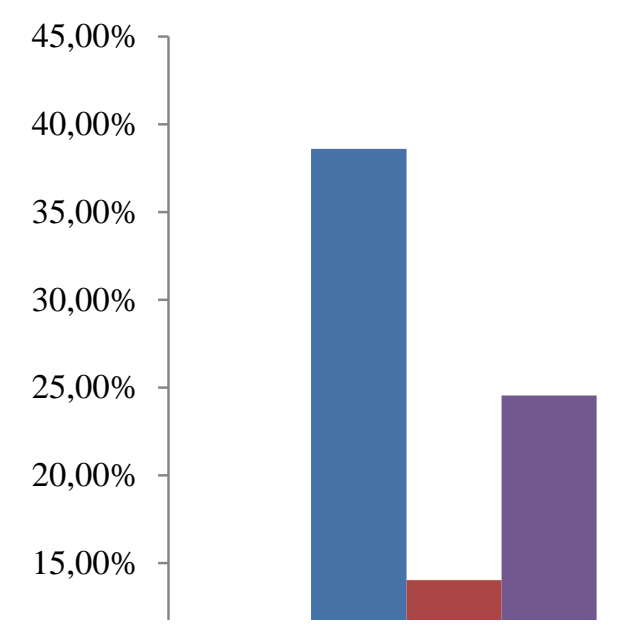

O gênero Aspergillus sp. (Figura 2) foi o de maior infestação na espécie da aroeira, com percentual representativo de 38,69\%, seguido de Fusarium sp. com $24,56 \%$. Os fungos filamentosos do gênero Aspergillus são fungos hialinos, saprófitos, encontrados no solo e na vegetação em decomposição e têm sido crescentemente, relatados em casos de infecções sistêmicas ou disseminados. A patogenicidade destes fungos oportunistas está na alta concentração dos microconídios no ar, facilitando a sua inalação (LOYOLA, 2006).
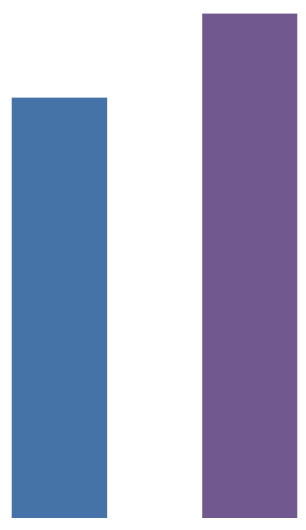

A citrinina é uma micotoxina oriunda do crescimento de várias espécies de fungos dos gêneros, tais como o Penicillium sp. e Aspergillus sp. O consumo de baixa concentração, por animais sensíveis às micotoxinas, pode conduzir à morte dentro de poucas horas. O mais comum é a ocorrência de intoxicações que variam de agudas a crônicas, afetando, principalmente os animais criados sob regime de confinamento, tais como aves e suínos (CARVALHO et al., 2005).

O Aspergillus sp. apresenta micotoxinas, que segundo Lança (2010), 
causa doença, a qual resulta do crescimento do fungo dentro das áreas previamente alteradas dos pulmões. A complicação mais temida nesta situação é a hemoptise volumosa - tosse com expectoração de sangue em grande quantidade, causando a morte.

A incidência de fungos detectados nas cascas indica a exposição das plantas medicinais a condições incorretas de armazenamento o que pode ocasionar doenças. Em Imperatriz, Maranhão, as plantas coletadas para análise, estavam expostas em condições irregulares, ou seja, eram encontradas em sacos plásticos com rasuras, no chão em contato com a umidade, em bancadas, levando assim a condições de riscos para a saúde humana.

Nos mercados, os materiais vegetais não apresentam padronização, ou seja, não são apresentados em embalagens e não apresentam rótulos.
O uso de material vegetal para automedicação é algo rotineiro e cultural em muitas localidades da região em pesquisa. A forma de armazenamento e acondicionamento do material pode acarretar em uma proliferação de microrganismos patogênicos à saúde humana. Microrganismos, especificamente os fungos, podem exalar toxinas $\mathrm{e}$ acometer doenças no trato digestivo e respiratório dos seres humanos. As qualidades sanitárias desses materiais relevam uma condição satisfatória para uso.

Figura 2. Aspergillus sp.: (a) isolado do material vegetal da aroeira (Myrocroduon urundeuva Allemão); (b) isolado do material vegetal da catuaba (Erythroxylum vacciniifolium).
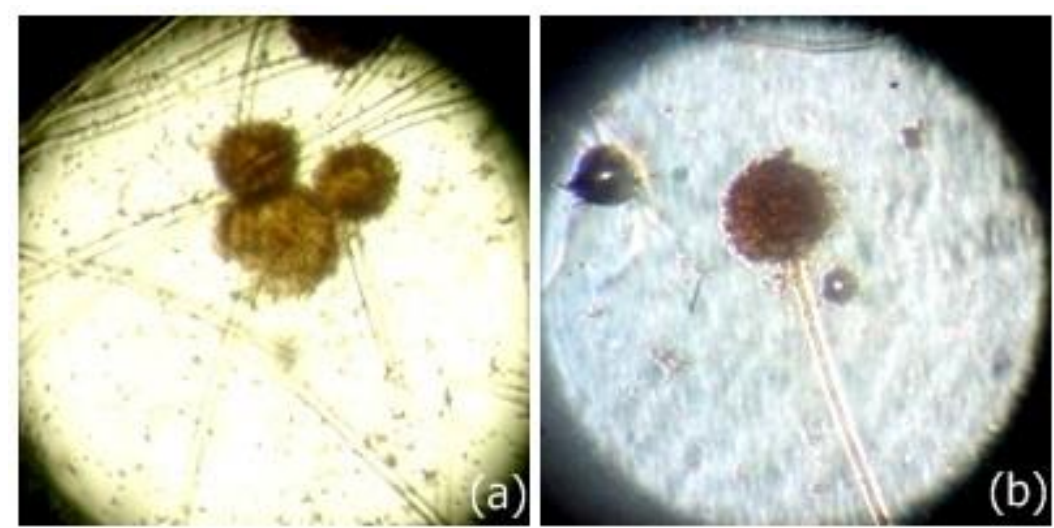
Foram identificados quatro gênero de fungos fitopatogênicos, dentre eles: Aspergillus sp., Fusarium sp., Penicillium sp., Rhizopus sp. Com o presente resultado da pesquisa, faz-se necessário que se estabeleçam medidas adequadas de controle higiênico-sanitário para garantir a qualidade e segurança deste tipo de produto desde a coleta, armazenamento, manipulação até o produto final (medicamentos caseiros ou fitoterápicos).

\section{REFERÊNCIAS}

ANGRA, M. F.; FRANÇA, P. F.; BARBOSA-FILHO, A. C. Synopsis of the plants known as medicinal and poisonous in Northeast of Brazil. Rev. Bras. Farmacogn, v. 17, n. 1, p.114-140, jan./mar. 2007.

BARNETT, H. L.; HUNTER, B. B. Illustrated Genera of Imperfect Fungi. 4th. ed. Saint Paul, MN : APS PRESS, 1998.

CAB International. Fusarium sp. Disponível em: <http://faem.ufpel.edu.br/ dfs/patologiasementes/cgi-bin/sementes/d etalhes.cgi?praga=96>. Acesso em: 23 abr. 2013.

CARVALHO C. A., FERNANDES, B. C. T. M. FREIRE, R. B. Supressão da resposta imunitária humoral causada pela citrinina. Arq. Bras. Med. Vet. Zootec., Belo Horizonte, v. 57, n. 2, p. 171-176, Abr. 2005.

CELESTE, M. L.; LILIANA J. G.; SILVA, A. S. Fumonisinas. Portugal: CEF - Universidade de Coimbra, 2004. 245p.

FREIRE, F. C. O.; VIEIRA, I. G. P.; GUEDES, M. I. F.; MENDES, F. N. P.
Micotoxinas: importância na alimentação e na saúde humana e animal. Fortaleza: Embrapa Agroindústria Tropical, 2007. 48p. (Embrapa Agroindústria Tropical. Documentos, 110).

LANÇA, M. A. Aspergilose Pulmonar. 2010. Disponível em: http://www.abcdas aude.com.br/artigo.php?39. Acesso em: 16 maio 2014.

LOYOLA, A. B. A. T. Avaliação da suscetibilidade de Aspergillus spp. e Fusarium spp. a antifúngicos por microdiluição em caldo e sistema de monitorização de crescimento de hifas, Campinas, 2006. 76 f. Tese (Doutorado em Ciências Médicas) - Universidade Estadual de Campinas, Faculdade de Ciências Médicas, Campinas, 2006.

PRADO, H. Aspergillus. 2 ed. São Paulo: UFMS, 2008.

SILVA, I.; FRANCO, S. L.; MOLINARI, S. L. Noções sobre o organismo humano e utilização de plantas medicinais. 3 ed. Cascavel (PR): Assoeste, 1995. 